\title{
Advanced Alignment of the ATLAS Inner Detector
}

\author{
Jonathan Stahlman, on behalf of the ATLAS Collaboration
}

Penn

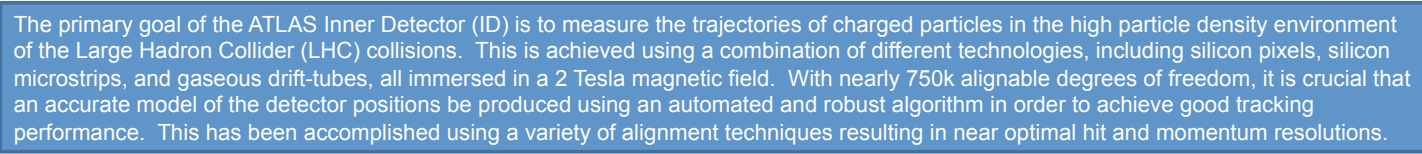

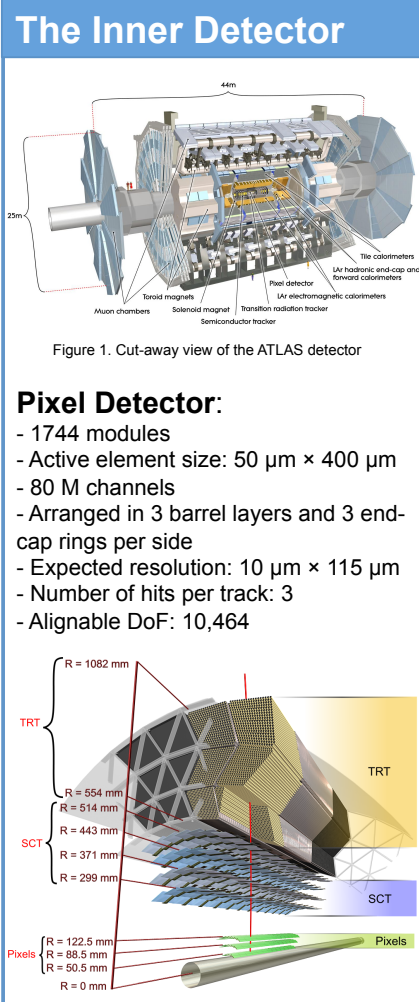

Figure 2. Cut-away view of the ID Barrel Geometry

\section{Semiconductor Tracker}

\section{(SCT):}

-4088 modules

- Active element size: $80 \mu \mathrm{m} \times 12 \mathrm{~cm}$ $6 \mathrm{M}$ channels

- Arranged in 4 barrel layers and 9 endcap rings per side

- Expected resolution: $17 \mu \mathrm{m} \times 580 \mu \mathrm{m}$ Number of hits per track: 8

Alignable DoF: 24,528

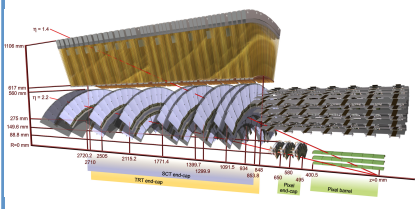

\section{Transition Radiation}

Tracker (TRT):

- 350,048 straw tubes

- Active element size: $4 \mathrm{~mm} \times 71 \mathrm{~cm}$

(barrel), $4 \mathrm{~mm} \times 39 \mathrm{~cm}$ (end-cap)

- Arranged in barrel modules (parallel to

LHC beam) and in end-cap wheels

(perpendicular to LHC beam)

Expected resolution: $130 \mu \mathrm{m}$

- Number of hits per track: 30

- Alignable DoF: 701,696

\section{Alignment Algorithm}

The alignment employs a track based alignment algorithm which seeks to minimize the track hit residuals. The residual is the distance from a recorded hit to an extrapolated track position within a detector element. To minimize the residuals, one constructs a $x^{2}$ as below, where $r(t, a)$ is the vector of residuals which depend on $\mathbf{t}$, the track parameters and $\mathbf{a}$, the alignment parameters. $\mathbf{V}$ is the covariance matrix of the hit residuals. Minimizing this $x^{2}$ and Taylor expanding the solution to first order around an initial residual $\mathbf{r}_{0}$ shows that a matrix inversion is needed to determine the changes $\delta \mathbf{a}$ to the alignment parameters.

\section{$\chi^{2}=\sum_{\text {tracks }} \mathbf{r}^{T}(\mathbf{t}, \mathbf{a}) V^{-1} \mathbf{r}(\mathbf{t}, \mathbf{a})$}

Minimization Condition

$\frac{d \chi^{2}}{d \mathbf{a}}=0 \Rightarrow 2 \sum_{\text {tracks }}\left[\left(\frac{d \mathbf{r}}{d \mathbf{a}}\right)^{T} V^{-1} \mathbf{r}\right]=0$

Taylor Expansion : $\mathbf{r}=\mathbf{r}_{0}+\frac{d \mathbf{r}}{d \mathbf{a}} \delta a$

$\underset{\sum_{\text {tracks }}\left[\left(\frac{d \mathbf{r}}{d \mathbf{a}}\right)^{T} V^{-1}\left(\frac{d \mathbf{r}}{d \mathbf{a}}\right)\right]}{\mathrm{N}_{\mathrm{DoF}} \text { matrix }} \delta a+\sum_{\text {tracks }}\left(\frac{d \mathbf{r}}{d \mathbf{a}}\right)^{T} V^{-1} \mathbf{r}_{0}=0$

\section{Global vs. Local $\mathbf{X}^{2}$}

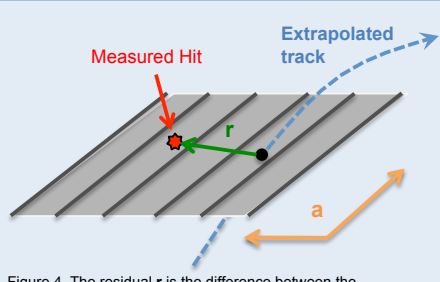

Figure 4. The residual $r$ is the difference between the
measured hit position and the extrapolated track position

The above Global $X^{2}$ algorithm is used to calculate the alignment parameters when the number of alignment parameters is relatively small, e.g. aligning the silicon only (35k degrees of freedom). However, given a very large number of alignment parameters (as in the TRT wire-by-wire alignment), the matrix inversion becomes computationally prohibitive and a simplification must be made. A local $x^{2}$ calculation is performed instead, which discards the correlations between alignable structures. The matrix to be inverted becomes block diagonal, greatly simplifying the necessary computations. The local $x^{2}$ algorithm must then be iterated to account for the correlations between the different alignable structures.
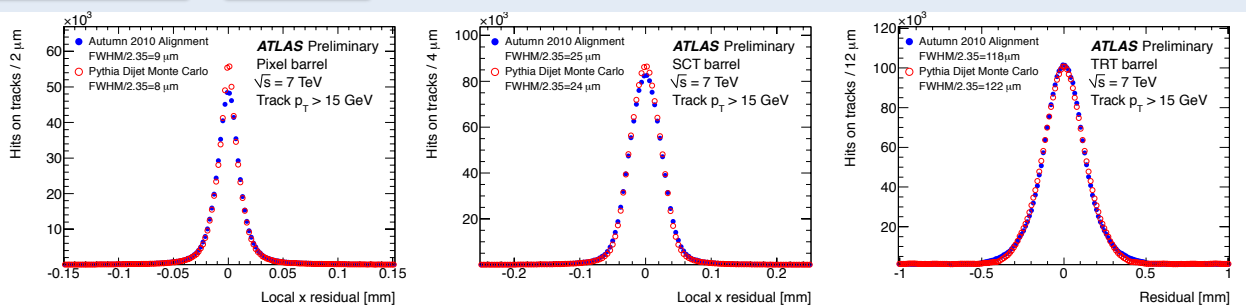

\section{Alignment Results}

The final alignment procedure uses a combination of the Global and Local $x^{2}$ residual minimizations. The hit resolution achieved in $7 \mathrm{TeV}$ data achieves similar performance to that of the simulation, which uses a perfectly aligned geometry.

\section{Systematic Misalignments}

The $x^{2}$ alignment cannot correct for all possible detector misalignments due to the presence of so called weak modes. A weak mode is a coherent detector deformation which biases the track parameters but leaves the $x^{2}$ unchanged. For example, the "curl" weak mode consists of systematic misalignments in $\phi$ as function of the detector element radius, as in Figure 6. A "curl" results in a charge-antisymmetric bias in the reconstructed momentum.

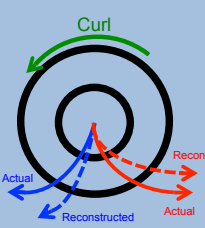

Figure 6. The

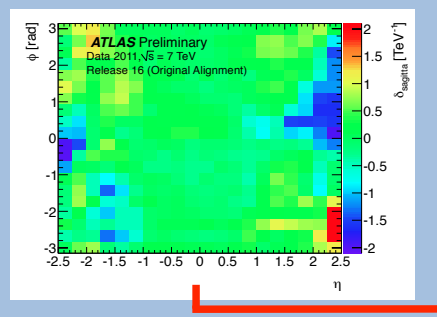

Figure 7. Measured momentum biases throughout the detector before a momentum constrained alignment. The fors

Correcting Weak Modes Weak modes can be corrected by applying an external constraint in the alignment algorithm. The track momentum was corrected using calorimeter information and the momentum was then fixed during the alignment procedure. As a result, the biases previously observed were significantly reduced, as in Figure 8 . This also resulted in a near optimal $Z$ mass resolution, as in Figure 9.

\section{Measuring the Effects of Weak Modes}

Weak modes causing track parameter biases can be detected by using external information about the track parameters, such as the energy deposited by electrons in the calorimeter or the invariant mass of a known particle resonance. In particular shifts in the reconstructed $\mathrm{Z}$ mass in $\mathrm{Z} \rightarrow \mu^{+} \mu^{-}$decays can be used to estimate the track momentum biases for muons. Parametrizing the momentum biases chargeantisymmetrically as in the equation below, the measured bias parameter $\delta_{\text {sagitta }}$ is plotted as a function of the muon pseudorapidity $(\eta)$ and $\phi$ in Figure 7.

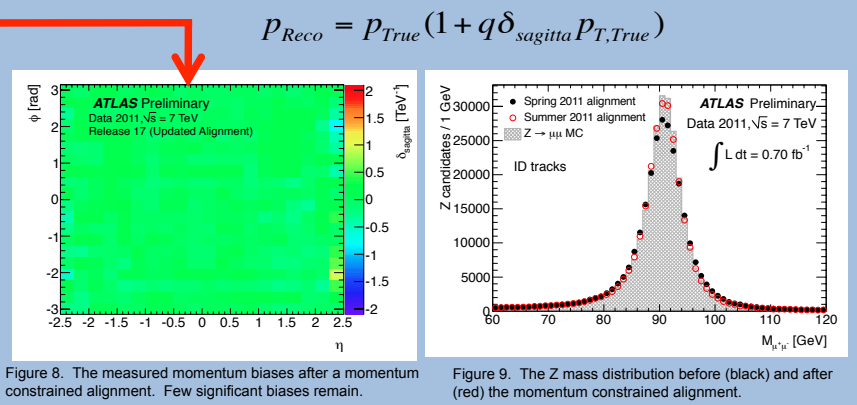

